\title{
Efeitos do Exercício Físico Sobre o Trato Gastrintestinal
}

\section{Effects of Physical Exercise on the Gastrointestinal Tract}

Claudio Andre Barbosa de Lira'

Rodrigo Luiz Vancini ${ }^{1}$

Antonio Carlos da Silva

Viviane Louise Andree Nouailhetas ${ }^{2}$

1. Centro de Estudos de Fisiologia do Exercício (CEFE) da Universidade Federal de São Paulo (UNIFESP)

2. Departamento de Biofísica da Universidade Federal de São Paulo (UNIFESP)

\section{Endereço para correspondência: \\ Antonio Carlos da Silva \\ Departamento de Fisiologia \\ Universidade Federal de São Paulo \\ Rua Botucatu, 862, $5^{\circ}$ andar \\ 04023-062 São Paulo, SP, Brazil \\ Tel: $+55-11-55764513$ \\ Fax: +55-11-55710171 \\ E-mail: carlos.antonio@unifesp.br}

Submetido em 21/09/2006

Versão final recebida em 31/10/2006

Aceito em 15/12/2006

\begin{abstract}
RESUMO
O impacto do exercício sobre o trato gastrintestinal (TGI), apesar de pouco investigado, é uma área de grande interesse. O exercício aeróbio intenso e de longa duração pode provocar sintomas gastrintestinais. Estes podem ser divididos em sintomas superiores (vômitos, náuseas e pirose retroesternal - azia) e inferiores (diarréia, cólica abdominal, perda de apetite, sangramento, aceleração dos movimentos intestinais e vontade de defecar). A etiologia desses sintomas durante o exercício é multifatorial e inclui a redução do fluxo sanguíneo intestinal, a liberação de hormônios gastrintestinais, o estresse mecânico sobre o TGl, a desidratação, os fatores psicológicos, a idade, o sexo, a dieta e o nível de treinamento do indivíduo. Por outro lado, o exercício de baixa intensidade tem efeito protetor sobre o TGl, principalmente com relação à predisposição a certas doenças como o câncer de cólon, a diverticulite, a colelitíase e a constipação. Diversos mecanismos são postulados para explicar os efeitos do exercício sobre o TGl, contribuindo para o desenvolvimento de estratégias terapêuticas no tratamento de indivíduos com sintomas e doenças gastrintestinais.
\end{abstract}

Palavras-chave: exercício, trato gastrintestinal, sintomas gastrintestinais e doenças gastrintestinais.

\begin{abstract}
The impact of exercise on the gastrointestinal tract (Gl tract), although being little investigated, is a field of high interest. Intense endurance aerobic exercise can lead to gastrointestinal symptoms. These symptoms can be classified into upper symptoms (vomiting, nausea and retrosternal pyrosis - heartburn) and lower symptoms (diarrhea, abdominal cramps, loss of appetite, bleeding, accelerated bowel transit time, urge to defecate). These symptoms' etiology during exercise is multifactorial and includes: reduction of intestinal blood flow, release of gastrointestinal hormones, mechanical stress on the Gl tract, dehydration, psychological factors, age, gender, diet, and training status. On the other hand, low intensity exercise has a protective effect on the GI tract; mainly, with certain diseases, such as, colon cancer, diverticular disease, cholelithiases, and constipation. A variety of mechanisms have been postulated to explain the effects of exercise on the Gl tract, contributing to the development of therapeutic strategies in the treatment of patients with gastrointestinal symptoms and diseases.
\end{abstract}

Keywords: exercise, gastrointestinal tract, gastrointestinal symptoms, and gastrointestinal diseases.

\section{INTRODUÇÃO}

Tradicionalmente, as pesquisas envolvendo a fisiologia do exercício se concentram nas respostas e adaptações dos sistemas respiratório, cardiovascular e muscular, já que muitos dos benefícios relacionados à saúde e qualidade de vida com a prática regular de exercício são decorrentes de respostas e adaptações nestes três sistemas orgânicos. Por outro lado, o exercício também exerce influência sobre outros sistemas que não estão diretamente relacionados com sua execução, tais como, o sistema imune ${ }^{(1,2)}$ e o trato gastrintestinal $(\mathrm{TGI})^{(3)}$.

Neste contexto, o impacto do exercício sobre o TGl, apesar de pouco explorado, é uma área de grande interesse. A partir do início da década de 80, vários pesquisadores passaram a investigar os efeitos do exercício sobre o TGl por meio de estudos clínicos e epidemiológicos, principalmente com relação aos sintomas maléficos ${ }^{(3,4)}$. Recentemente, entretanto, tal interesse tem sido focado sobre os potenciais benefícios do exercício sobre o TGl, tais como a diminuição da prevalência de câncer de cólon e da constipação(4-6). Outro aspecto interessante e, pouco explorado, é o efeito do exercício sobre a reatividade intestinal. Recentemente, demonstramos, por meio de uma abordagem celular e funcional, que tanto o exercício agudo como o crônico alteram a reatividade do íleo ${ }^{(7-10)}$. Sendo assim, o presente estudo teve como objetivo revisar os principais efeitos maléficos e benéficos do exercício no TGl e seus potenciais impactos sobre a saúde.

\section{Efeitos maléficos do exercício sobre o TGI}

O exercício predominantemente aeróbio e de longa duração, como aquele executado por maratonistas, triatletas e ciclistas, pode provocar sintomas gastrintestinais. A maioria dos trabalhos afirma que de 20 a 50\% da população praticante de esportes de longa duração apresenta pelo menos um sintoma ${ }^{(11)}$. Estes são divididos em sintomas superiores (vômitos, náuseas e pirose retroesternal - azia) e inferiores (diarréia, cólica abdominal, perda de apetite, sangramento, aceleração dos movimentos intestinais e vontade de defecar) $)^{(6,12-17)}$.

A etiologia dos sintomas gastrintestinais durante o exercício é multifatorial e inclui a redução do fluxo sanguíneo intestinal, a liberação de hormônios gastrintestinais ${ }^{(11,18-24)}$, o estresse mecânico sobre o TGI ${ }^{(25,26)}$, a

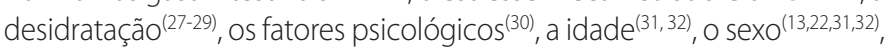
a dieta ${ }^{(22,23,33,34)}$ e o nível de treinamento do indivíduo ${ }^{(23)}$.

Comentaremos a seguir as principais causas para o desenvolvimento de sintomas gastrintestinais em praticantes de exercícios de longa duração. 


\section{Redução do fluxo sanguíneo intestinal}

A redução do fluxo sanguíneo intestinal durante o exercício é decorrente da vasoconstrição do leito vascular esplâncnico pela ação das catecolaminas sobre os receptores $\alpha$-adrenérgicos ${ }^{(35)}$ e/ou da ativação do sistema renina-angiotensina pela hipovolemia induzida pelo exercício decorrente da desidratação( ${ }^{(36-42)}$. Tal redução pode atingir níveis críticos, e ser agravada durante condições de hipertermia, desidratação, hipoglicemia, hipóxia, alterações da viscosidade sanguínea, deformabilidade e agregabilidade dos eritrócitos ou pela combinação destes fatores ${ }^{(5,23,43-45)}$. Em particular, Rehrer et a ${ }^{(27,28)}$ verificaram que a perda de 3,5 a 4,0\% do peso corporal provocada pela desidratação durante o exercício está associada ao aumento da incidência de sintomas gastrintestinais.

No repouso, 90\% do fluxo sanguíneo para o intestino é dirigido para a mucosa ${ }^{(46)}$. Portanto, esta região é a mais susceptível para apresentar alterações em conseqüência da redução do fluxo sanguíneo intestinal durante o exercício ${ }^{(24,35)}$. É sabido que a mucosa do TGI age como uma barreira entre os meios externo e interno, evitando a penetração de compostos antigênicos, carcinogênicos e tóxicos da luz intestinal para o fluido intersticial ${ }^{(24,47-49)}$. Por outro lado, o comprometimento da mucosa pela redução do fluxo sanguíneo provocada pelo exercício aumenta a permeabilidade intestinal através das junções comunicantes (estruturas protéicas que permitem a passagem de íons e moléculas entre células justapostas). A passagem de compostos agressivos através das junções comunicantes estimula a migração de neutrófilos ${ }^{(48-52)}$, que quando ativados disparam uma resposta imune local com geração de radicais livres e liberação de enzimas lisossomais, danificando ainda mais o epitélio intestinal(49,53). Essa seqüência de eventos contribui para potencializar o aumento da permeabilidade intestinal e da resposta inflamatória, ocasionando o desenvolvimento de sintomas gastrintestinais e, em alguns casos, até endotoxemia(49,52,54-56).

Também já foi demonstrado que atletas praticantes de atividades de longa duração, especialmente os ultramaratonistas, podem sofrer sangramento intestinal durante e após o exercício ${ }^{(12,57-60)}$. Esta situação é decorrente da diminuição da oferta de oxigênio e nutrientes em conseqüência da redução do fluxo sanguíneo intestinal o que pode produzir desarranjo morfológico e funcional da mucosa e necrose de células gástricas ${ }^{(61)}$, hepatócitos ${ }^{(62)}$ e células intestinais ${ }^{(61,63-36)}$. Embora tal perda de sangue pelo TGI seja transitória, pode causar perda clinicamente importante de ferro e anemia ${ }^{(4,66-68)}$.

\section{Hormônios gastrintestinais}

Muitos hormônios associados com a função do TGI no repouso, responsáveis pela secreção, absorção e motilidade, têm suas concentrações plasmáticas alteradas durante o exercício e podem colaborar para o desenvolvimento de sintomas gastrintestinais. Entre eles podemos citar a colecistocinina, o peptídeo intestinal vasoativo (VIP), a secretina, o polipeptídeo pancreático, a somatostatina, o peptídeo histidina-metionina (PHM), o peptídeo YY, o peptídeo inibitório gástrico, a gastrina, o glucagon, a motilina, as catecolaminas, as endorfinas e as prostaglandinas ${ }^{(23,69-71)}$.

O VIP, O PHM, a gastrina e a motilina são liberados em altas quantidades pelo intestino isquêmico(19,24). Estes peptídeos diminuem a absorção de íons sódio e aumentam o conteúdo intestinal, promovendo diarréia. Além disso, a secretina, o glucagon, o peptídeo inibitório gástrico e as prostaglandinas também são liberados na circulação sanguínea em decorrência do exercício exaustivo e são conhecidos por aumentar a secreção intestinal e, desse modo, causar diarréia ${ }^{(23,24)}$.

A diarréia também pode resultar do relaxamento do cólon ocasionado pela queda da concentração plasmática de insulina e pelo aumento dos níveis de polipeptídeo pancreático. Concomitantemente, ocorre diminuição da pressão exercida pelo músculo do esfíncter interno, resultante do aumento do PHM e VIP (24). Além do mais, o tônus parassimpático durante o exercício moderado pode aumentar o trânsito intestinal por estimular a contração da musculatura lisa intestinal ${ }^{(12)}$. Contrariando essa teoria, existe $\mathrm{o}$ fato destes sintomas gastrintestinais serem prevalentes em exercícios aeróbios e intensos, onde o tônus simpático é dominante, causando o relaxamento da musculatura lisa ${ }^{(12,24)}$.

\section{Fatores mecânicos}

A estimulação mecânica sobre o TGI produzida pelo impacto proporcionado pelo exercício pode contribuir para o desenvolvimento de sintomas gastrintestinais, já que quando a mucosa intestinal é distendida e/ou friccionada libera VIP e prostaglandinas causando aumento da secreção intestinal e diarréia. Isto é corroborado pelo fato da freqüência desses sintomas ser aproximadamente duas vezes superior durante a corrida em comparação com o ciclismo e a natação, onde o impacto e a vibração mecânicos são menores ${ }^{(25,26,72)}$. Além disso, já foi demonstrado que a hipertrofia do músculo psoas pode causar pressão contra o cólon e estimular o aumento da motilidade e da defecação(22).

\section{Fatores psicológicos}

A interação entre o cérebro e o intestino tem recebido muita atenção nas últimas décadas ${ }^{(73)}$, pois a incidência de sintomas gastrintestinais relacionados ao estresse emocional têm sido descrita desde o início do século passado ${ }^{(74)}$. Já foi demonstrado que o estresse emocional diminui o tempo de trânsito orocecal( ${ }^{(75)}$ e aumenta a atividade colônica ${ }^{(76)}$. A presença de sintomas gastrintestinais em muitas situações tem sido atribuída a causas psicogênicas, tal como, a ansiedade e o estresse emocional(77). Segundo Sullivan, Wong ${ }^{(13)}, 32 \%$ dos atletas que se queixam de sintomas gastrintestinais, apresentam quadros de estresse emocional.

\section{Outras causas}

Outras causas para o aparecimento de sintomas gastrintestinais incluem a dieta, o gênero, a idade e o estado de treinamento do indivíduo.

Quanto à dieta, a ingestão de altas quantidades de suco de laranja, comidas gordurosas, proteína, vitamina C, bebidas hipertônicas de carboidratos, alimentos ricos em fibras e grandes refeições antes de competição predispõem à manifestação de sintomas gastrintestinais (22,23,33). Muito embora existam relatos que o consumo de cafeína antes do exercício possa aumentar a queixa de sintomas gastrintestinais, Van Nieuwenhoven et $a^{(34)}$ recentemente mostraram que as queixas dos sintomas gastrintestinais foram maiores em atletas que consumiram solução de carboidratos durante o exercício em comparação com a ingestão de água. Entretanto, quando se adicionou cafeína na solução de carboidrato, a queixa de sintomas gastrintestinais não foi diferente daquela comparada à solução de carboidrato.

Com relação ao gênero, a maioria dos trabalhos mostrou que os

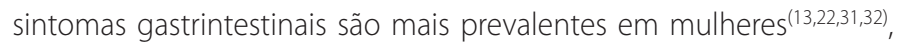
especialmente no período menstrual. A idade também é um fator que influencia a incidência dos sintomas gastrintestinais, já que os atletas mais jovens e menos experientes são os mais acometidos, dado a menor vivência de treinamento(31,32).

Finalmente, quanto ao estado de treinamento, os indivíduos destreinados ou atletas que retornam ao treinamento após um período de inatividade apresentam maior incidência de sintomas gastrintestinais, principalmente com treinamento de alta intensidade ${ }^{(23)}$. 


\section{Tratamento e prevenção}

Algumas medidas podem otimizar a digestão e a absorção dos alimentos durante o exercício e reduzir a ocorrência dos sintomas gastrintestinais(23).

1. Aumentar apropriadamente o volume e a intensidade do treinamento;

2. Planejar o regime de hidratação durante as sessões de treinamento e a competição;

3. Evitar o consumo de soluções hipertônicas de carboidratos;

4. Evitar o consumo de dieta rica em fibras antes da competição;

5. Ingerir gorduras e proteínas com moderação antes da competição;

6. Evitar altas doses de vitamina $\mathrm{C}$ e bicarbonato;

7. Defecar e urinar antes do exercício;

8. Evitar o uso de drogas que atuem sobre o sistema gastrintestinal, tais como os antiinflamatórios não esteroidais;

9. Realizar exame gastroenterológico, caso haja queixa freqüente de sintomas gastrintestinais em repouso e durante o exercício.

\section{Efeitos benéficos do exercício sobre o TGI}

O exercício, principalmente o de baixa intensidade, tem efeito protetor sobre o TGl. Evidências recentes obtidas em nosso laboratório mostraram que a prática habitual de atividade física moderada protege o íleo de camundongos contra os efeitos do envelhecimento ${ }^{(8)}$. 0 envelhecimento em camundongos produz espessamento da camada muscular do íleo, associado à desorganização parcial das mitocôndrias, entretanto nos animais exercitados, o envelhecimento não foi capaz de produzir tais efeitos histológicos ${ }^{(8)}$.

Alguns estudos indicam uma relação inversa entre a atividade física e as doenças do TGI tais como, o câncer de cólon ${ }^{(78-81)}$, a diverticulite ${ }^{(82)}$, a colelitíase (cálculo na vesícula biliar) ${ }^{(83,84)}$ e a constipação(85).

Em relação ao câncer de cólon, existem evidências consistentes de que homens e mulheres fisicamente ativos apresentam uma redução de cerca de 50\% do risco de desenvolverem câncer de cólon ${ }^{(4,78,80,86,87)}$. Esta redução acontece independente de outros fatores de risco, tais como a dieta e a composição corporal. A maioria dos autores sugere que existe uma relação dose-resposta já que foi demonstrado que o treinamento intenso confere maior proteção que o moderado ${ }^{(4,80,88)}$. Por exemplo, no trabalho de Demarzo(89), foi demonstrado que o cólon de ratos submetidos ao exercício crônico apresentou redução estatisticamente significante na resposta hiperproliferativa a um agente carcinogênico em comparação ao cólon de animais do grupo controle. Em contrapartida, o exercício exaustivo aumentou significativamente tal resposta ${ }^{(90)}$.

Um dos mecanismos postulado para a queda da prevalência de câncer de cólon pela atividade física é a redução do tempo de trânsito intestinal o que diminuiria o tempo de contato entre a mucosa do cólon e os possíveis compostos carcinogênicos presentes no conteúdo intestinal( ${ }^{(80,87)}$. Além do mais, a atividade física modula positivamente alguns fatores relacionados com o desenvolvimento do câncer de cólon, tais como, a disfunção do sistema imune, a dieta, a obesidade, a ação da insulina, os níveis de prostaglandinas e os triglicérides, os estoques de ferro e os mecanismos de defesa antioxidante ${ }^{(4,80,88)}$.

Poucos estudos foram realizados em relação ao desenvolvimento de câncer em outros órgãos do TGl. Os estudos que envolveram o binômio exercício e câncer de estômago não chegaram a uma conclusão comum $^{(91)}$, como também não foi demonstrada uma relação entre exercício e prevenção de câncer de pâncreas ou reto(81-92). Até onde sabemos, não existem estudos para avaliar o impacto do exercício sobre o desenvolvimento do câncer de esôfago, ducto e vesícula biliar.
Assim como para o câncer de cólon, o exercício exerce um efeito protetor contra o desenvolvimento de colelitíase (cálculo biliar) ${ }^{(83,84)}$, existindo uma clara relação dose-resposta entre atividade física e prevenção da colelitíase. Os mecanismos pelos quais o exercício pode modificar a patogênese da colelitíase são poucos conhecidos, porém a diminuição da secreção de colesterol biliar e o aumento da motilidade da vesícula biliar e do cólon são fatores postulados para a prevenção da colelitíase ${ }^{(93)}$. Além disso, fatores como a tolerância à glicose, a concentração de insulina, os triglicérides e os hormônios reguladores da vesícula biliar, tais como a colecistocinina e os níveis baixos de lipoproteína de baixa densidade (LDL), são alterados pelo exercício e podem modificar favoravelmente a patogênese dessa doença( ${ }^{(93,94)}$.

Manousos et $a^{(95)}$ sugeriram que a atividade física poderia reduzir o risco de diverticulite, por mostrarem que esta patologia é mais prevalente entre pessoas sedentárias do que em pessoas fisicamente ativas. Mais recentemente, Aldoori et al ${ }^{(82)}$ mostraram uma relação inversa entre a atividade física e o surgimento de diverticulite em um estudo de coorte em 47.678 pessoas durante quatro anos. $O$ mecanismo postulado para a influência da atividade física na patogênese dessa doença é a diminuição do tempo de trânsito intestinal promovido pela atividade física(82,96-100).

Por fim, alguns estudos mostraram uma relação inversa entre constipação e atividade física ${ }^{(77,85,101)}$. Karam, Nies ${ }^{(102)}$ mostraram melhora do quadro de constipação e redução do uso de laxantes após a combinação de um programa de atividade física associada com dieta, porém o efeito do exercício isoladamente não foi avaliado. No entanto, o resultado desse estudo não foi confirmado por Meshkinpour et al (103) que não encontraram diferença entre o estado de constipação antes e após o treinamento aeróbio de baixa intensidade em 8 pacientes constipados. Assim, os resultados dos estudos são divergentes de tal modo que o exercício parece exercer um efeito sobre a constipação, porém as evidências ainda não são conclusivas.

Os prováveis mecanismos para os benefícios do exercício sobre o TGI incluem a alteração da motilidade do cólon, a diminuição do fluxo sanguíneo intestinal, o estresse mecânico produzido pela corrida sobre o intestino, a compressão do cólon pela musculatura abdominal e o aumento da ingestão de fibras como resultado do aumento do gasto energético ${ }^{(104,105)}$. É importante ressaltar que grande parte dos fatores responsáveis pelos benefícios do exercício sobre o TGl são também os responsáveis pelos malefícios, portanto, existe uma relação doseresposta e um limiar de intensidade que separa os dois efeitos sobre o TGI.

\section{CONCLUSÃO}

O efeito do exercício sobre a fisiologia humana tem recebido muita atenção nas últimas décadas, mas o TGI tem sido negligenciado. 0 exercício produz uma variedade de efeitos sobre o TGl que podem ter impacto negativo e/ou positivo. Neste contexto, diversos mecanismos são postulados para explicar os efeitos maléficos e benéficos do exercício sobre o TGl, tais como, a diminuição do fluxo sanguíneo intestinal, o estresse mecânico, a desidratação, a mudança no tempo de trânsito intestinal e a modulação do eixo neuro-imuno-endócrino. A combinação desses mecanismos, portanto, pode explicar os potenciais efeitos do exercício sobre o TGl e, desse modo, contribuir para o desenvolvimento de estratégias terapêuticas para minimizar possíveis efeitos deletérios e/ou atuar como adjuvante no tratamento de indivíduos com doenças gastrintestinais.

Todos os autores declararam não haver qualquer potencial conflito de interesses referente a este artigo. 


\section{REFERÊNCIAS BIBLIOGRÁFICAS}

1. Pedersen BK, Hoffman-Goetz L. Exercise and the immune system: regulation, integration, and adaptation. Physiol Rev 2000; 80: 1055-81.

2. Petersen AM, Pedersen BK. The anti-inflammatory effect of exercise. J Appl Physiol 2005; 98: 1154-62.

3. Casey E, Mistry DJ, MacKnight JM. Training room management of medical conditions: sports gastroenterology. Clin Sports Med 2005; 24: 525-40.

4. Peters HP, de Vries WR, van Berge-Henegouwen GP, Akkermans LM. Potential benefits and hazards of physical activity and exercise on the gastrointestinal tract. Gut 2001; 48: 435-9.

5. Gisolfi CV. Is the GI System Built For Exercise? News Physiol Sci 2000; 15: 114-9.

6. Simren M. Physical activity and the gastrointestinal tract. Eur J Gastroenterol Hepatol 2002: 14: 1053-6.

7. De Lira CA. Efeitos do exercício moderado sobre os mecanismos de transduçăo de sinal em íleo isolado de camundongos C57BL/6. São Paulo, 2004 (Tese de Mestrado apresentada à Universidade Federal de São Paulo/Escola Paulista de Medicina).

8. Rosa EF, Silva AC, Ihara SS, Mora OA, Aboulafia J, Nouailhetas VL. Habitual exercise program protects murine intestinal, skeletal, and cardiac muscles against aging. J Appl Physiol 2005; 99: 1569-75.

9. Vancini RL. Relação entre o exercício aeróbio moderado e o estresse oxidativo induzido pelo peróxido de hidrogênio $\left(\mathrm{H}_{2} \mathrm{O}_{2}\right)$ sobre a resposta contrátil de íleo isolado de camundongo. São Paulo, 2005 (Tese de Mestrado apresentada à Universidade Federal de São Paulo/Escola Paulista de Medicina).

10. De Lira CA, Vancini RL, Ihara SS, da Silva AC, Aboulafia J, Nouailhetas VL. Aerobic exercise affects C57BL/6 murine intestinal contractile function. Eur J Appl Physiol. 2008 Feb 14; [Epub ahead of print].

11. Gil SM, Yazaki E, Evans DF. Aetiology of running-related gastrointestinal dysfuction. How far is the finishing line? Sports Med 1998; 26: 365-78.

12. Butcher JD. Runner's diarrhea and other intestinal problems of athletes. Am Fam Phys 1993; 48: 623-7.

13. Sullivan S, Wong C, Heidenheim P. Does running cause gastrointestinal symptoms? A survey of 93 randomly selected runners compared with controls. N Z Med J 1994; 107: 328-31.

14. Peters HP, Bos M, Seebregts L, Akkermans LMA, Van Berge Henegouwen GP, Bol E, et al. Gastrointestinal symptoms in long-distance runners, cyclists, and triathletes: prevalence, medication, and etiology. Am J Gastroenterol 1999a; 94: 1570-81

15. Peters HP, Zweers M, Backx FJ, Bol E, Hendriks ER, Mosterd WL, de Vries WR. Gastrointestinal symptoms during long-distance walking. Med Sci Sports Exerc 1999b; 31: 767-73.

16. Kondo T, Nakae Y, Mitsui T, Kagaya M, Matsutani Y, Horibe H, et al. Exercise-induced nausea is exaggerated by eating. Appetite 2001; 36: 119-25.

17. Van Nieuwenhoven MA, Brouns F, Brummer RJ. Gastrointestinal profile of symptomatic athletes at rest and during physical exercise. Eur J Appl Physiol 2004; 91: 429-34.

18. Brandsborg O, Christensen NJ, Galbo M, Brandsborg M, Lovgreen NA. The effect of exercise, smoking and propanolol on serum gastrin in patients with duodenal ulcer and in vagotomized subjects. Scan J Clin Lab Invest 1978; 38: 441-6.

19. Modlin I, Bloom S, Mitchell S. Plasma vasoactive intestinal polypeptide (VIP) levels and intestinal ischaemia. Experientia 1978; 34: 535-6.

20. Hilsted J, Galbo M, Sonne B, Schwarz T, Fahrenkrug J, de Muckadell OB, et al. Gastroenteropancreatic hormonal changes during exercise. Am J Physiol 1980; 239: 136-40.

21. Sullivan S, Champion M, Christofides N, Adrian T, Bloom S. Gastrointestinal regulatory peptide responses in long-distance runners. Phys Sports Med 1984; 12: 77-82

22. Riddoch C, Trinick T. Gastrointestinal disturbances in marathon runners. Br J Sports Med 1988; 22: 71-4.

23. Brouns F, Beckers E. Is the gut an athletic organ? Digestion, absorption and exercise. Sports Med 1993; 1:66-77.

24. Berg A, Müller HM, Rathmann S, Deibert P. The gastrointestinal system, an essential target organ of the athlete's health and physical performance. Exerc Immunology Rev 1999; 5: 78-95.

25. Sullivan S. Exercise-associated symptoms in triathletes. Phys Sports Med 1987; 15: 105-10.

26. Peters HP, Akkermans LM, Bol E, Mosterd WL. Gastrointestinal symptoms during exercise. The effect of fluid supplementation. Sports Med 1995; 20:65-76.

27. Rehrer NJ, Janssen GM, Brouns F, Saris WH. Fluid intake and gastrointestinal problems in runners competing in a 25-km race and a marathon. Int J Sports Med 1989; 10:22-5.

28. Rehrer NJ, Beckers EJ, Brouns F, ten Hoor F, Saris WH. Effects of dehydration on gastric emptying and gastrointestinal distress while running. Med Sci Sports Exerc 1990; 22: 790-5.

29. Van Nieuwenhoven MA, Vriens BE, Brummer RJ, Brouns F. Effect of dehydration on gastrointestinal function at rest and during exercise in humans. Eur J Appl Physiol 2000; 83: 578-84

30. Halvorsen FA, Ritland S. Gastrointestinal problems related to endurance event training. Sports Med 1992; 14: 157-63.

31. Keeffe E, Lowe D, Goss J, Wayne R. Gastrointestinal symptoms of marathon runners. West J Med 1984; 141: 481-4.

32. Worobetz L, Gerrard D. Gastrointestinal symptoms during exercise in enduro athletes: prevalence and speculations on the aetiology. NZ Mec J 1985; 98: 6444-6.

33. Shi X, Horn MK, Osterberg KL, Stofan JR, Zachwieja JJ, Horswill CA, Passe DH, Murray R. Gastrointestinal discomfort during intermittent high-intensity exercise: effect of carbohydrate-electrolyte beverage. Int J Sport Nutr Exerc Metab 2004; 14: 673-83.

34. Van Nieuwenhoven MA, Brouns F, Kovacs EM. The effect of two sports drinks and water on Gl complaints and performance during an 18-km run. Int J Sports Med 2005; 26: 281-5.

35. Granger DN Richardson PD, Kvietys PR, Mortillaro NA. Intestinal blood flow Gastroenterol 1980: 78: 837-63.

36. Bulkley GB, Kvietys PR, Parks DA, Perry MA, Granger DN. Relationship of blood flow and oxygen consumption to ischemic injury in the canine small intestine. Gastroenterology 1985; 89: 852-7.

37. Bailey RW, Bulkley GB, Hamilton SR, Morris JB, Smith GW. Pathogenesis of nonocclusive ischemic colitis. Ann Surg 1986; 203: 590-9.

38. Luger A, Deuster PA, Debolt JE, Loriaux DL, Chrousos GP. Acute exercise stimulates the renin-angiotensinaldosterone axis: adaptive changes in runners. Horm Res 1988; 30: 5-9.

39. Suvannapura A, Levens NR. Local control of mesenteric blood flow by the renin-angiotensin system. Am J Physiol 1988; 255: 267-74

40. Ferrari R, Ceconi C, Rodella A, De Giuli F, Panzali A, Harris P. Temporal relations of the endocrine response to exercise. Cardioscience 1991; 2: 131-9.

41. Aldigier JC, Huang H, Dalmay F, Lartigue M, Baussant T, Chassain AP, et al. Angiotensin-converting enzyme inhibition does not suppress plasma angiotensin II increase during exercise in humans. J Cardiovasc Pharmacol 1993; 21: 289-95.

42. Wilcox MG, Howard TJ, Plaskon LA, Unthank JL, Madura JA. Current theories of pathogenesis and treatment of nonocclusive mesenteric ischemia. Dig Dis Sci 1995; 40: 709-16.

43. Vandewalle $H$, Lacombe C, Lelievre JC, Poirot C. Blood viscosity after a 1-h submaximal exercise with and without drinking. Int J Sports Med 1988; 9: 104-7.

44. Kam LW, Pease WE, Thompson PD. Exercise-related mesenteric infarction. Am J Gastroenterol 1994; 89: 1899-900

45. Otte JA, Oostveen E, Geelkerken RH, Groeneveld AB, Kolkman JJ. Exercise induces gastric ischemia in healthy volunteers: a tonometry study. J Appl. Physiol 2001; 91: 866-71.

46. Chou CC, Grassmick B. Motility and blood flow distribution within the wall of the gastrointestinal tract. Am J Physiol 1978; 235: 34-9.

47. Hollander D. The intestinal permeability barrier. Scand J Gastroenterol 1992; 27: 721-6.

48. Bjarnason I. Intestinal permeability. Gut 1994; 1(Suppl): S18-22.

49. Pals KL, Chang RT, Ryan AJ, Gisolfi CV. Effect of running intensity on intestinal permeability. J Appl Physiol 1997; 82: 571-6.

50. Rumage J, Astanisz K, Scicchitano R, Hunt RH, Perdue MH. Effect of immunologic reactions on rat intestinal epithelium. Gastroenterology 1988; 94: 1368-75.

51. Somasundaram S, Hayllar H, Rafi S, Wrigglesworth JM, Macpherson AJS, Bjarnason I. The bioquemical basis of nonsteroidal anti-inflammatory drug-induced damage to the gastrointestinal tract: a review and a hypothesis. Scand I Gastroenterol 1995; 30: 289-99.

52. Lambert GP, Broussard L, Mason BL, Mauermann WJ, Gisolfi CV. Gastrointestinal permeability during exercise: effects of aspirin and energy-containing beverages. J Appl Physiol 2001; 90: 2075-80.

53. Baska RS, Moses FM, Graeber G, Kearney G. Gastrointestinal bleeding during an ultramarathon. Dig Dis Sci 1990; 35: 270-6.
4. Smetanka RD, Lambert GP, Murray R, Eddy D, Horn M, Gisolfi CV. Intestinal permeability in runners in the 1996 Chicago marathon. Int I Sport Nutr 1999: 9:426-33.

55. Jeukendrup AE, Vet-Joop K, Sturk A, Stegen JH, Senden J, Saris WH, et al. Relationship between gastrointestinal complaints and endotoxaemia, cytokine release and the acute-phase reaction during and after a long-distance triathlon in highly trained men. Clin Sci 2000; 98: 47-55.

56. Lambert GP. Role of gastrointestinal permeability in exertional heatstroke.Exerc Sport Sci Rev 2004; 32: 185-90.

57. Halvorsen FA, Lyng J, Ritland S. Gastrointestinal bleeding in marathon runners. Scand J Gastroenterol 1986; $21: 493-7$.

58. Cooper BT, Douglas SA, Firth LA, Hannagan JA, Chadwick VS. Erosive gastritis and gastrointestinal bleeding in a female runner. Prevention of the bleeding and healing of the gastritis with H2-receptor antagonists. Gastroenterology 1987; 92: 2019-23.

59. Lehair P, Bigard MA. Prevalence of occult digestive hemorrhages during heavy-endurance exercise. Gastroenterol Clin Biol 1993; 17: 773.

60. Peters HP, Wiersma WC, Akkermans LM, Bol E, Kraaijenhagen RJ, Mosterd WL, et al. Gastrointestinal mucosal integrity after prolonged exercise with fluid supplementation. Med Sci Sports Exerc 2000; 32: 134-42.

61. Sato N, Kamada T, Shichiri M, Kawano S, Abe H, Hagihara B. Measurement of hemoperfusion and oxygen sufficiency in gastric mucosa in vivo. Evidence of mucosal hypoxia as the cause of hemorrhagic shockinduced gastric mucosal lesion in rats. Gastroenterology 1979; 76: 814-9.

62. Gores GJ, Flarsheim CE, Dawson TL, Nieminen AL, Herman B, Lemasters JJ. Swelling, reductive stress, and cell death during chemical hypoxia in hepatocytes. Am J Physiol 1989; 257: 347-54.

63. Kingham JG, Whorwell PJ, Loehry CA. Small intestinal permeability. 1. Effects of ischaemia and exposure to acetyl salicylate. Gut 1976; 17: 354-61.

64. Chiu CJ, Scott HJ, Gurd FN. Intestinal mucosal lesion in low-flow states. II The protective effect of intraluminal glucose as energy substrate. Arch Surg 1970; 101: 484-8.

65. Parks DA, Grogaard B, Granger DN. Comparison of partial and complete arterial occlusion models for studying intestinal ischemia. Surgery 1982; 92: 896-901.

66. McMahon LF Jr, Ryan MJ, Larson D, Fisher RL. Occult gastrointestinal blood loss in marathon runners. Ann Intern Med 1984; 100: 846-7.

67. Lampe JW, Slavin JL, Apple FS. Iron status of active women and the effect of running a marathon on bowel function and gastrointestinal blood loss. Int J Sports Med 1991; 12:173-9.

68. Rudzki SJ, Hazard H, Collinson D. Gastrointestinal blood loss in triathletes: it's etiology and relationship to sports anaemia. Aust J Sci Med Sport 1995; 27: 3-8.

69. Demers LM, Harrison TS, Halbert DR, Santen RJ. Effect of prolonged exercise on plasma prostaglandin levels. Prostaglandins Med 1981; 6: 413-8.

70. O'Connor AM, Johnston CF, Buchanan KD, Boreham C, Trinick TR, Riddoch CJ. Circulating gastrointestinal hormone changes in marathon running. Int J Sports Med 1995; 16: 283-7.

71. Banfi G, Marinelli M, Bonini P, Gritti I, Roi GS. Pepsinogens and gastrointestinal symptoms in mountain marathon runners. Int J Sports Med 1996: 17: 554-8.

72. Rehrer NJ, Meijer GA. Biomechanical vibration of the abdominal region during running and bicycling. J Sports Med Phys Fitness 1991; 31: 231-4.

73. Mulak A, Bonaz B. Irritable bowel syndrome: a model of the brain-gut interactions. Med Sci Monit 2004; 10; RA55-62

74. Cannon WB. The movements of the intestines studied by means of the Roentgen rays. Am J Physiol 1902:6:251-77.

75. O'Brien JD, Thompson DG, Day SJ, Burnham WR, Walker E. Perturbation of upper gastrointestinal transit and antroduodenal motility by experimentally applied stress: the role of beta-adrenoreceptor mediated pathways. Gut 1989: 30: 1530-9.

76. Barone FC, Deegan JF, Price WJ, Fowler PJ, Fondacaro JD, Ormsbee HS 3rd. Cold-restraint stress increases rat fecal pellet output and colonic transit. Am J Physiol 1990; 258: G329-37.

77. Sullivan SN, Wong C. Runners' diarrhea. Different patterns and associated factors. J Clin Gastroenterol 1992; 14: 101-4.

78. Colditz GA, Cannuscio CC, Frazier. Physical activity and reduced risk of colon cancer: implications for prevention. Cancer Causes Control 1997; 8: 649-67.

79. Oliveira SA, Christos PJ. The epidemiology of physical activity and cancer. Ann NY Acad Sci 1997; 833: 79-90.

80. Quadrilatero J, Hoffman-Goetz L. Physical activity and colon cancer (A systematica review of potential mechanisms). J Sports Med Phys Fitness 2003; 43:121-38.

81. World Cancer Research Fund/American Institute for Cancer Research. Food, Nutrition, and the prevention of cancer: a global perspective 1997; 216-24

82. Aldoori WH, Giovannucci EL, Rimm EB, Ascherio A, Stampfer MJ, Colditz GA, et al. Prospective study of physical activity and the risk of symptomatic diverticular disease in men. Gut 1995; 36: 276-82.

83. Leitzmann MF, Rimm EB, Willett WC, Spiegelman D, Grodstein F, Stampfer MJ, Colditz GA, Giovannucci E. Recreational physical activity and the risk of cholecystectomy in women. N Engl J Med 1999; 341:777-84.

84. Leitzmann MF, Giovannucci EL, Rimm EB, Stampfer MJ, Spiegelman D, Wing AL, Willett WC. The relation of physical activity to risk for symptomatic gallstone disease in men. Ann Intern Med 1998; 128: 417-25.

85. Everhart JE, Go VL, Johannes RS, Fitzsimmons SC, Roth HP, White LR. A longitudinal survey of self-reported bowel habits in the United States. Dig Dis Sci 1989; 34: 1153-62.

86. Haydon AM, Macinnis R, English D, Giles G. The effect of physical activity and body size on survival after diagnosis with colorectal cancer. Gut 2006: 55: 62-7.

87. Shephard RJ, Shek PN. Associations between physical activity and susceptibility to cancer: possible mechanisms. Sports Med 1998; 26: 293-315.

88. Slattery ML. Physical activity and colorectal cancer. Sports Med 2004; 34: 239-52

89. Demarzo MM. Efeitos da atividade física em marcadores biológicos da carcinogênese química do cólon de ratos Wistar. Ribeirăo Preto, 2005 (Tese de Doutorado apresentada à Faculdade de Medicina de Ribeirão Preto da Universidade de São Paulo).

90. Demarzo MM, Garcia SB. Exhaustive physical exercise increases the number of colonic preneoplastic lesions in untrained rats treated with a chemical carcinogen. Cancer Lett 2004; 216: 31-4.

91. Lee IM. Physical activity, fitness, and cancer. In: Bouchard C, Shephard RJ, Stephens T. Physical activity, fitness, and health. Champaign(IL):Human Kinetics; 1994. p.814-31.

92. Lee IM, Paffenbarger RSJ. Physical activity and its relation to cancer risk: a prospective study of college alumni. Med Sci Sports Exerc 1994; 26: 831-7.

93. Erpecum van K, Van Berge-Henegouwen GP. Gallstones: an intestinal disease? Gut 1999: 44: 435-8.

94. Bennion $\perp$, Grundy SM. Risk factors for the development of cholelithiasis in man. N Engl J Med 1978; 299: 1161-7.

95. Manousos ON, Vrachliotis G, Papaevangelou G, Detorakis E, Doritis P, Stergiou L, Merikas G. Relation of diverticulosis of the colon to environmental factors in Greece. Am J Dig Dis 1973; 18: 174-6.

96. Keeling WF, Martin BJ. Gastrointestinal transit during mild exercise. J Appl Physiol 1987; 63: 978-81.

97. Martin BJ. Gut transit with exercise. Gastroenterology 1990; 99: 290.

98. Keeling WF, Harris A, Martin BJ. Loperamide abolishes exercise-induced orocecal liquid transit acceleration. Dig Dis Sci 1993; 38: 1783-7.

99. Bingham SA, Cummings JH. Effect of exercise and physical fitness on large intestinal function. Gastroenterology 1989; 97: 1389-99.

100. Coenen C, Wegener M, Wedmann B, Schmidt G, Hoffmann S. Does physical exercise influence bowel transit time in healthy young men? Am J Gastroenterol 1992; 87: 292-5.

101. Kinnunen O. Study of constipation in a geriatric hospital, day hospital, old people's home and at home. Aging 1991; 3: 161-70.

102. Karam SE, Nies DM. Student/staff collaboration: a pilot bowel management program. J Gerontol Nurs 1994; 20: 32-40.

103. Meshkinpour H, Kemp C, Fairshter R. Effect of aerobic exercise on mouth-to-cecum transit time. Gastroenterology 1998; 96: 938-41.

104. Oettle GJ. Effect of moderate exercise on bowel habit. Gut 1991; 32: 941-4

105. Koffler KH, Menkes A, Redmond RA, Whitehead WE, Pratley RE, Hurley BF. Strength training accelerates gastrointestinal transit in middle-aged and older men. Med Sci Sports Exerc 1992; 24: 415-9. 